\title{
In vitro transition of Schistocephalus solidus (Cestoda) from coracidium to procercoid and from procercoid to plerocercoid
}

\author{
P.J. Jakobsen ${ }^{\text {a,b,* }}$, J.P. Scharsack ${ }^{\text {b,c }}$, K. Hammerschmidt ${ }^{\text {b,d }}$, P. Deines ${ }^{\text {e,f }}$, M. Kalbe ${ }^{\text {b }}$, M. Milinski ${ }^{\text {b }}$ \\ a Institute for Biology, University of Bergen, Thor Møhlensgt. 55, 5020 Bergen, Norway \\ ${ }^{\mathrm{b}}$ Max Planck Institute for Evolutionary Biology, Department of Evolutionary Ecology, August Thienemann Str. 2, 24306 Plön, Germany \\ ${ }^{\mathrm{c}}$ Animal Evolutionary Ecology Group, Institute for Evolution and Biodiversity, University of Muenster, Huefferstr. 1, 48149 Muenster, Germany \\ ${ }^{\mathrm{d}}$ NZ Institute for Advanced Study, Massey University, Private Bag 102904, Auckland, New Zealand \\ e Max Planck Institute for Limnology, Department of Physiological Ecology, August Thienemann Str. 2, 24306 Plön, Germany \\ ${ }^{\mathrm{f}}$ School of Biological Sciences, The University of Auckland, Private Bag 92019, Auckland, New Zealand
}

\section{A R T I C L E I N F O}

\section{Article history:}

Received 16 February 2010

Received in revised form 16 September 2011

Accepted 19 September 2011

Available online 15 October 2011

\section{Keywords:}

Schistocephalus solidus

In vitro

Procercoid

Plerocercoid

Tegumental layer

Defence

Trade-off

\begin{abstract}
A B S T R A C T
With the present study, a culture system for successive life-cycle stages of the tapeworm Schistocephalus solidus was developed and this report documents for the first time, cultivation of the procercoid stage of $S$. solidus from eggs. Additionally we have transformed procercoids dissected from experimentally infected copepods and cultured procercoids into the early plerocercoid stage in vitro. Observations in the culture suggest that the coracidia can interact with their external environment and need no host specific stimuli, except for the components in the culture medium, for activation and hatching from the embryophore. Increasing the culture medium $\mathrm{pH}$ from 7.3 to 8.0 improved escape rates and frequencies of hook contractions, suggesting that the oncosphere may recognize and respond to environmental conditions along the host intestine. Procercoids in the culture did not stop growing indicating that conditions within the copepod may be important to limit growth and to induce transformation to plerocercoids. When procercoids are dissected from copepods and transferred to the culture, the outer tegument layers and cercomer starts to loosen. Comparison of the lectin staining of the loosened outer tegument layers and cercomer in procercoids dissected from copepods confirms that transitions of both, the oncosphere to procercoid and procercoid to plerocercoids, has taken place in the in vitro cultures.
\end{abstract}

(c) 2011 Elsevier Inc. All rights reserved.

\section{Introduction}

The tapeworm Schistocephalus solidus is a hermaphrodite with a complex life-cycle involving a cyclopoid copepod as first host, followed by the three-spined stickleback, Gasterosteus aculeatus, and finally a homeothermic host, most often a bird (Smyth, 1946). The parasites' eggs are expelled into water with the final host's faeces, where embryos start to develop, if the temperature is high enough. After 3 weeks at $18{ }^{\circ} \mathrm{C}$, coracidia, the first larval stage, hatch. To hatch, the egg needs to be illuminated (Dubinina, 1966), probably because the coracidia produce a light-released enzyme to open the egg operculum through which the ciliated coracidium creeps out (Smyth and McManus, 1989). The coracidium has a brief life span, and has to be ingested by the first intermediate host within $96-120 \mathrm{~h}$ at $5-8{ }^{\circ} \mathrm{C}$ (Dubinina, 1966) or $24-36 \mathrm{~h}$ at $18^{\circ} \mathrm{C}$ (Wedekind, 1997). In the copepod's gut the oncosphere (the larva contained within the embryophore) is thought to escape from the

* Corresponding author at: Institute for Biology, University of Bergen, Thor Møhlensgt. 55, 5020 Bergen, Norway. Fax: +47 (0) 55584401

E-mail address: Per.Jakobsen@bio.uib.no (P.J. Jakobsen). inner and outer cavity of the inner egg envelope, the embryophore, before it penetrates the copepod's gut and enters its body cavity. Here, the larva grows and develops into the procercoid. When fully developed the infective procercoid is characterized by a cercomer, a structure of unknown function. Just after ingestion of the infected copepod by the stickleback host, the procercoid "switches coats" by losing its outer surface coat and the cercomer, most often in the frontal part of the stickleback intestine (Hammerschmidt and Kurtz, 2007). The third larval stage, the plerocercoid, develops and grows to become infective to the definitive host after several months (Hopkins and Smyth, 1951; Clarke, 1954). Schistocephalus sp. are characterized by a long-lasting plerocercoid stage in the fish intermediate host (Dubinina, 1966; Chervy, 2002). In terms of host involvement, the plerocercoid has been the most studied phase in the life-cycle, as the parasite has long-term effects on the host, often with detrimental impact on the health, fecundity, and behaviour of infected fish (Dubinina, 1966; Arme and Owen, 1967; Milinski, 1985, 2006; Smyth and McManus, 1989; Barber and Huntingford, 1995; Barber and Arnott, 2000; Barber and Scharsack, 2010). Although life-cycle stages are usually studied in isolation, recent research indicates that state of the tapeworms in their 
copepod host, seems to have a significant impact on the life history traits of the parasites in their second host (Hammerschmidt and Kurtz, 2005a; Hammerschmidt et al., 2009). Fitness of the parasite is hence determined by a cascading effect of its transition through its host spectrum (Parker et al., 2009).

S. solidus has developed into a natural model to study the ecology and evolutionary questions of host-parasite interactions (Hammerschmidt and Kurtz, 2007, 2009 and references therein) and complex life-cycles (Parker et al., 2003, 2009; Hammerschmidt et al., 2009). Many questions address the life-cycle as a whole, and hence all stages of the parasite come into focus. The parasite is characterized by taking most of its energy from its fish host. Maturation is triggered by increasing temperature to $40{ }^{\circ} \mathrm{C}$ and the tapeworm will reach sexual maturity after $36 \mathrm{~h}$ (Smyth, 1954). Because the plerocercoid can easily be removed sterile from the coelom of the stickleback, it was the first cestode successfully matured in vitro to produce fertile eggs (Smyth, 1946). This early in vitro breeding of $S$. solidus gave rise to a large number of studies on the basic biology, biochemistry, and physiology of this parasite (Smyth, 1990). This was due to the fact that culturing techniques allow experiments to be carried out, under controlled reproducible conditions, free from interference by host-related factors (Smyth, 1990). Cultures are also important for general parasitology studies because it may be possible to track different developmental stages or transition processes of parasites within their natural hosts. However, younger stages of S. solidus have only been investigated in their natural hosts. Extensions of in vitro cultures to S. solidus's complete life-cycle would therefore provide a powerful tool, as it would allow investigation of its different stages under rigorous experimental conditions.

Although there have been attempts (Smyth, 1959), transformation from eggs to fully developed procercoids, have, to our knowledge (see also Smyth and McManus, 1989) never been successfully conducted in Diphyllobothridea nor Bothriocephalidea. Transmission to the plerocercoids has however, earlier been successfully conducted in Spirometra mansoni (Berntzen and Mueller, 1964), but not in S. solidus.

\section{Materials and methods}

\subsection{Transition of coracidia to procercoids: the artificial copepod}

Infected sticklebacks, were caught during autumn 2006 from the lake Skogseidvatnet $60^{\circ} 13^{\prime} \mathrm{N}^{\circ} 53^{\prime} \mathrm{E}$, Western Norway, and brought to the Max Planck Institute for Evolutionary Biology in Plön, Germany. Here the fish were kept in large tanks and fed ad libitum. The tapeworms were dissected aseptically from the sticklebacks under sterile conditions and bred in an in vitro system that replaces the bird's gut (Smyth, 1946; Wedekind, 1997). Tapeworms were bred in size-matched pairs to avoid selfing (Lüscher and Milinski, 2003). Eggs were then allowed to develop in sterile filtered tap water for 3 weeks at $18{ }^{\circ} \mathrm{C}$ in the dark. In the evening before use, they were exposed to light for hatching (Dubinina, 1966).

Newly hatched coracidia in water were diluted $1: 10$ with $18^{\circ} \mathrm{C}$ culture medium (for medium composition, see Table 1 ), or $18^{\circ} \mathrm{C}$ sterile tap water and $200 \mu \mathrm{l}$ aliquots were transferred to wells of 96-well flat bottom microtitre plates. The cultures were incubated in a moist chamber in dark, with $2 \% \mathrm{CO}_{2}$ at $18{ }^{\circ} \mathrm{C}$. Three times per week, $50 \%(\mathrm{v} / \mathrm{v})$ of the culture supernatant was replaced by fresh medium (Table 1). Larvae were monitored, using an inverted microscope at $200 \times$ magnification. The frequency of coracidia transformed to oncospheres and surviving untransformed coracidia (number out of 20 counted per well) were recorded in culture
Table 1

Additives to Minimum Essential Medium (MEM, Sigma M2279) used for cultivation of procercoid and plerocercoid stages (culture medium).

\begin{tabular}{lll}
\hline Ingredients & Catalogue No. & Final concentration \\
\hline L-Glutamin & Sigma G7513 & $10 \mathrm{mmol} \mathrm{l}^{-1}$ \\
Antibiotic, antimycotic & Sigma A 5955 & $10 \mathrm{ml} \mathrm{l}^{-1}$ \\
Glucose & Roth X997.2 & $1.5 \mathrm{~g} \mathrm{l}^{-1}$ \\
Yeast extract & Becton Dickinson 212750 & $5 \mathrm{~g} \mathrm{l}^{-1}$ \\
Sodium bicarbonate $^{\text {Fetal bovine serum }}{ }^{\mathrm{a}}$ & Merck 1.06329.05000 & $5.04 \mathrm{~g} \mathrm{l}^{-1}(\mathrm{pH} \mathrm{7.3)}$ \\
Chicken serum $^{\mathrm{a}}$ & Sigma F4135 & $10 \% \mathrm{v} / \mathrm{v}$ \\
\hline
\end{tabular}

For cultures with higher $\mathrm{pH}$, the medium was titrated to $\mathrm{pH} 8.0$ with $1 \mathrm{~mol} \mathrm{l}^{-1}$ $\mathrm{NaOH}$ solution. Note that the $\mathrm{pH}$ referred to is based on fresh medium.

a Heat inactivated.

after $48 \mathrm{~h}$ in culture media with $\mathrm{pH} 7.3$ and 8.0. Transformation rates were compared to coracidia incubated in sterile tap water.

The number of hook contractions per minute in transformed oncospheres, was counted in the two $\mathrm{pH}$ regimes $2 \mathrm{~h}$ after the coracidia were introduced to the culture medium.

\subsection{Transformation of procercoids to plerocercoids: the artificial stickleback}

The first intermediate hosts, Macrocyclops albidus copepods, were cultured and exposed to one coracidium each in the laboratory as described previously (van der Veen and Kurtz, 2002). The culture-population originated from 20 individuals from lake Skogseidsvatnet, Norway, the habitat of origin of the parasites. Instars ranging from the $\mathrm{C} 5$ copepodite stage and older were used as hosts for the procercoids. Copepods were kept at $20^{\circ} \mathrm{C}$ and 16:8 light: dark cycle. For infection, they were kept singly in wells of a 24-well microtitre plate and starved for 2 days before exposure to the parasite. Coracidia from three different parasite pairs were used for infection. Fourteen days post-infection, procercoids were dissected out of the copepods. Infected copepods were rinsed in sterile filtered water, placed under the microscope, and anaesthetized with carbonated water with added antibiotics $(200 \mu \mathrm{g} / \mathrm{ml}$, Sigma A 5977). After $2 \mathrm{~min}$, the carbonated water was replaced by sterile water. The abdomen of the copepod was removed to release the procercoids into water, which were then transferred into the culture medium with a pipette. To evaluate the effect of the anaesthization, we also conducted 10 dissections using sterile filtered instead of carbonated water. Cultured procercoids were regularly inspected with an inverted microscope.

\subsection{Lectin labelling of surface layers}

To evaluate the development of the parasites in culture, we analyzed their surface carbohydrate composition by using lectin labelling. In S. solidus, the different developmental stages can easily be distinguished by the composition of their surface carbohydrates: the surface of procercoids is dominated by PNA (Arachis hypogaea) binding sugars (ß-galactose-1,3 N-acetylgalactosamine (GalNAc) and D-galactose), whereas plerocercoids exhibit relatively more WGA (Triticum vulgaris) binding sugars ( $N$-acetylglucosamine (GlcNAc) and sialic acid residues) on their surface (Hammerschmidt and Kurtz, 2005b).

We compared the relative lectin binding intensity from procercoids that developed in culture (after 14 days: $N=5$ and after 33 days: $N=33$ ) with the ones that were directly dissected out of the copepod at 14 days post-infection $(N=20)$ and with those, which were dissected out of the copepod at 14 days post-infection, and put into culture (for $7-14$ days; $N=19$ ). As further reference, we used data on relative lectin binding intensity from 7-day-old plerocercoids $(N=31)$, published by Hammerschmidt and Kurtz 
(2005b). Each parasite was processed and labelled as described in Hammerschmidt and Kurtz (2005b), except that here TRITClabelled PNA and FITC-labelled WGA were used. As a control, lectins were incubated with their respective inhibitory ligands, which successfully blocked the labelling of the parasites (see Hammerschmidt and Kurtz, 2005b for more detail). To quantify the intensity of labelling, parasites were examined using an epifluorescence inverted microscope (Leica DM IRB) with the respective filters set to detect TRITC and FITC emission wavelengths (Hammerschmidt and Kurtz, 2005b). Images were obtained and analysed with the image analysis program IP Lab 3.6.2 (for Macintosh OS 9.2.2, Scanalytics, Inc.). The threshold function was used to identify the outline of the parasite, and the mean intensities of this area (scaled from 0 to 255) of the appropriate colour channel (red for TRITC, green for FITC) were obtained. The relative lectin labelling was calculated as previously described (Hammerschmidt and Kurtz, $2005 b)$, i.e. $100 \times$ intensity of $\mathrm{WGA} /(\mathrm{PNA}+\mathrm{WGA})$ gives the 'relative WGA labelling'.

Additionally, both 20 cultured and 20 in vivo bred procercoids were exposed to $2 \%$ bile extract diluted with sterile filtered tap water and inspected under an inverted microscope. The lengths of time before cercomers detached from procercoids were recorded.

\subsection{Data analysis}

Differences in oncosphere escape rates from their embryophores, hook contractions of coracidia transformed to oncospheres and the survival of newly transformed oncospheres were tested, with Kruskal-Wallis tests with culture method (media with the two pHs and sterile tap water) as effect variables.

To check whether the different stages of the tapeworms (culture only (14 and 33 days)), procercoids from copepods (14 days), procercoids and culture (14 days in copepod and 7 and 14 days in culture), plerocercoids ( 7 days in fish)) differed in their relative intensity of lectin labelling, we calculated an analysis of variance (ANOVA) with the relative labelling intensity of WGA $(100 \times$ WGA/WGA + PNA) as the response variable and parasite stage as effect. All analyses were performed with the JMP Version 5.0.1.2 $\left(\mathrm{SAS}^{\mathrm{TM}}\right)$ software for Macintosh ${ }^{\mathrm{TM}}$.

\section{Results}

\subsection{The artificial copepod, a culture system for S. solidus procercoids}

While no escape from the embryophore or activation was observed in tap water, almost all oncospheres within the coracidia were activated in the medium and a significant number managed to hatch from the embryophore. Escape rates from embryophores were also highest in the medium with pH $8.0(20.33 \%, N=30)$, lower at $\mathrm{pH} 7.3(6.61 \%, N=28)$, and nearly absent in sterile tap water $(0.16 \%, N=32)$ (Kruskal-Wallis $\chi_{2}^{2}=49.221, P<0.0001$ ). Moreover, hook contractions per minute of newly activated preoncospheres were significantly higher in culture medium with pH $8.0(9.75, N=20)$ compared to $\mathrm{pH} 7.3(0.8, N=20$, KruskalWallis $\left.\chi_{1}^{2}=23.453, P<0.0001\right)$. Finally, survival of coracidia was significantly higher when activated in the culture medium $(95.38 \%, N=39)$ compared to tap water $(9.64 \%, N=14)$ after $48 \mathrm{~h}$ (Kruskal-Wallis $\chi_{1}^{2}=33.554, P<0.0001$ ).

In the present study, coracidia containing activated preoncospheres, showed repetitive contractions and extensions, but continued swimming. Cilia beats in the coracidia ceased, however, as soon as the larvae started to penetrate the embryophores (Fig. 1).

Both procercoid growth and development in the cultures were slower than in the copepod host. Cercomer formation occurred much later in the in vitro cultures (27 days) than in the copepod M. albidus (9-11 days) kept at the same temperatures (Fig. 2). However, the procercoids continued to grow so that their final size exceeds the sizes found in natural copepod infections. Procercoids up to $1.5 \mathrm{~mm}$ in length have been observed after 183 days in the culture (Fig. 3 ).

\subsection{Transformation of procercoid to plerocercoids}

When procercoids were dissected out of the copepod, their activity increased and they started to extend and compress their bodies vigorously. When introduced to the culture medium, the procercoids (Fig. 4) started loosening their outer surface, before shedding it together with the cercomer (Fig. $4 a$ and b). The time procercoids took to escape from their outer layer in the culture, varied from a few minutes to 7 days. When exposing the procercoids of both the cultured and the in vivo produced procercoids with four drops of a $2 \%$ bile solution from perch Perca fluviatilis, time to shedding varies from $40 \mathrm{~s}$ to less than $3 \mathrm{~min}$. There was no significant difference in time used, before the procercoids produced in the culture or in vivo, had lost their cercomers $(P=0.49$, Wilcoxon rank sum test, two tailed, $W=43, N 1$ and $N 2=20$ ). The cultured procercoids was not observed to loose their cercomers at all. We also observed that in bile solution, the outer lectin layer and the cercomer seems to dissolve and disappear instead of being shed.

\subsection{Lectin labelling of the different parasite stages}

We found the different stages of the tapeworms (culture only, procercoids from copepods, procercoids placed into culture, plerocercoids) to significantly differ in their relative intensity of lectin binding (WGA: $F_{4,91}=23.454, P<0.0001$; Fig. 5). When comparing the different stages, the surface composition of the procercoids grown in culture for 14 days and those dissected from copepods did not differ significantly (Tukey-Kramer, post hoc, $P>0.05$ ). They were both mainly dominated by PNA binding carbohydrates as also found by Hammerschmidt and Kurtz (2005b). The procercoids that were cultured for 33 days did not possess a significantly different surface than the parasites that were dissected out of the copepod and then put into culture for 7-14 days (Tukey-Kramer post hoc test, $P>0.05$ ). Both dominated in PNA binding carbohydrates as previously observed by Hammerschmidt and Kurtz (2005b). Procercoids cultured from the egg for 33 days did not possess a significantly different surface than the parasites dissected out of the copepod and then put into culture for 7-14 days (Tukey-Kramer post hoc test, $P>0.05$ ). The procercoids that were cultured for 33 days clearly increased in the relative content of WGA binding carbohydrates over time and appeared to have made the transition to a more plerocercoid like surface.

\section{Discussion}

We here established a culture aiming to replace the first and the second intermediate host of a parasite with a complex life-cycle, $S$. solidus, and evaluated conditions which trigger the transformation between the parasite's developmental stages. Replacement of the final host (endothermic vertebrates, most often a bird) of S. solidus by an in vitro system has previously been established (Smyth, 1946). Furthermore, growth of small plerocercoids obtained from sticklebacks, the second intermediate host, has been conducted earlier in cultures and plerocercoids were grown to larger sizes and matured in vitro (Sinha and Hopkins, 1967). For the first time, we succeeded in generating procercoid-like worms from coracidia and early staged plerocercoids from procercoids dissected out of 

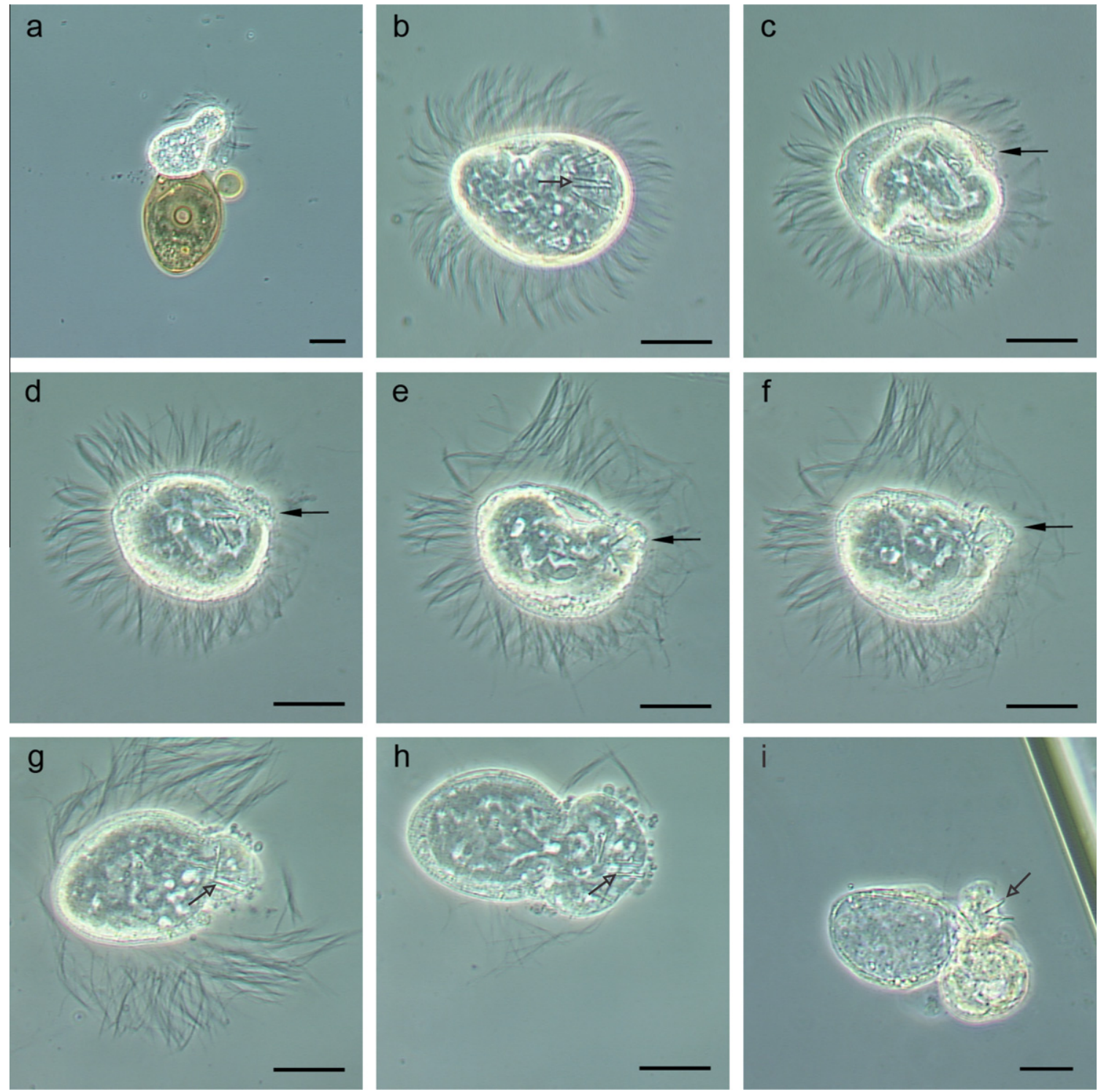

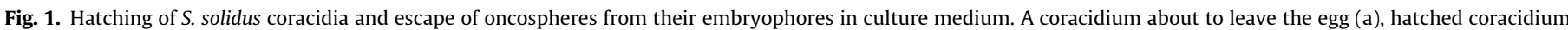

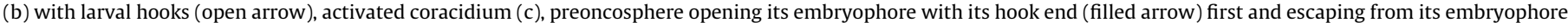
(d, e, f, g, and h), and an oncosphere escaping out of the embryophore, showing hook contractions (i) (each bar $=10 \mu \mathrm{m})$.

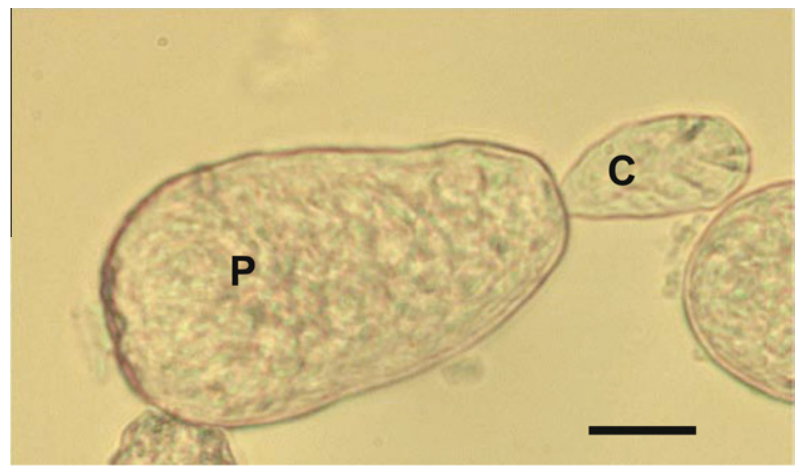

Fig. 2. A fully developed procercoid (P) bred for 33 days in the culture. The cercomer $(C)$ seems to be fully separated from the body (bar $=10 \mu \mathrm{m}$ ).

copepods and from cultured procercoids. Transformation of all stages in the life-cycle of $S$. solidus has now been achieved in vitro.
The S. solidus transformation process observed in our culture did not significantly differ from e.g. that of the shark tapeworm Lacistorhynchus semifasciata (Voge and Edmunds, 1969), when leaving their embryophores. After transfer of the coracidium from water into the culture medium, the preoncosphere of $S$. solidus lysed and/or ruptured an opening into the embryophore with its hook end, and crept out. This escape process was slow in some cases, as was the struggle to leave their outer surfaces in their transition from procercoids to plerocercoids. In the cultures, both processes might be more difficult, due to a lack of mechanical influence by their natural hosts. A more alkaline $\mathrm{pH}$ clearly increased the efficacy of the oncosphere-procercoid transformation; the larvae were more active, especially with respect to hook contractions and faster or more frequent escapes from their embryophores. This observation is in accordance with Polzer and Conradt (1994), who showed that procercoids of S. solidus had a collagenolytic activity with a $\mathrm{pH}$ optimum at 8.0. This $\mathrm{pH}$ optimum may be expected to coincide with the site of penetration in the copepod intestine, which is a critical phase for the parasite (van 


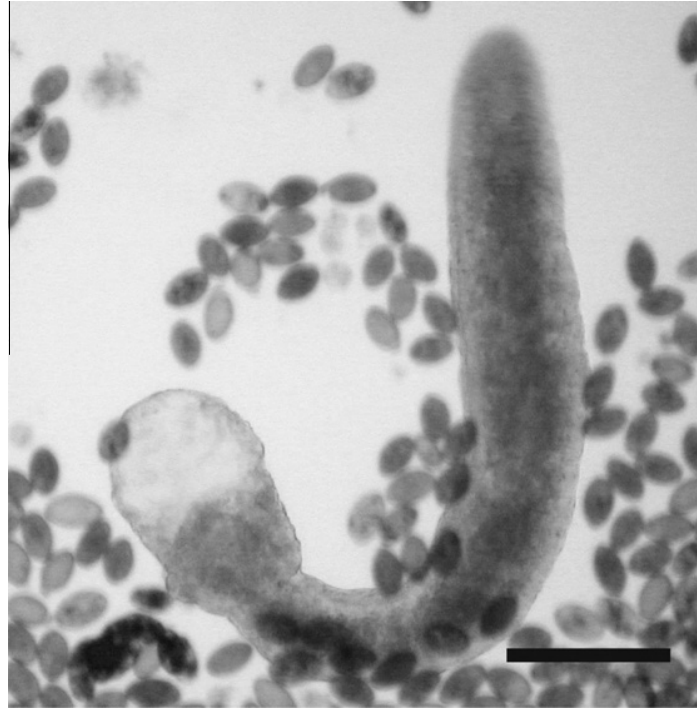

Fig. 3. Procercoid, cultured for 183 days, with an estimated length of $1.5 \mathrm{~mm}$ (bar $=100 \mu \mathrm{m}$ )

der Veen and Kurtz, 2002). In the only study describing the pH in the alimentary channel of copepods, Pond et al. (1995) found that the fore-intestine produces acids and that $\mathrm{pH}$ increased from neutral to $\mathrm{pH} 8.5$ in the mid and hind parts of the gut of unfed Calanus helgolandicus. Defaye et al. (1985) observed that mid and hind-gut of $M$. albidus contains cells producing enzymes for extra- and intracellular digestion. Thus, the newly escaped oncosphere in the copepod intestine is situated in an environment with both dissolved nutrients and a high $\mathrm{pH}$, similar to the conditions we were able to mimic in the cultures. If the parasite can achieve energy-uptake in the intestine, it might increase resources and add available time to foster the critical penetration process.

The most probable source of energy in the coracidium is the anaerobic consumption of reserve substances in the embryophore, as older coracidia have a drop in ATP, phospholipids and polysaccharides (Smyth and McManus, 1989). Coracidia survived significantly longer in our cultures, compared to water. Potential reasons for this could well be that the activation of the oncosphere enables them to take up nutrients and/or they have reduced energy expenditure due to osmoregulation. The activation of the oncosphere within the embryophore also shows that a chemical interaction between the oncosphere and the surrounding chemical environment is possible through the ciliated epithelial coat. Escape from their embryophore is improved after the mechanical rupture of the coracidia shell (embryophore outer surface) by the copepod (Dubinina, 1966). Because this is lacking in the cultures, time and energy needed for the escape process is probably increased in the in vitro cultures. Uptake of extra-nutrients from the culture medium might, however, support the oncosphere with extra nutrients and make the prolonged accomplishment of the escape process possible in the artificial culture situation.

Development of the procercoids in the culture was slower than in its copepod host and the time needed to develop a cercomer about twice as long as in the copepod host at the same temperature. This indicates that culture conditions do not exactly resemble the situation in the respective hosts and could be improved. Nevertheless, procercoids from the culture and those grown in the copepod had similar carbohydrate composition on their surface, indicating that they have started to develop vital structures.

When left for half a year in the culture, procercoids in the culture grew to sizes up to $1.5 \mathrm{~mm}$. The presence of a cercomer in these larvae indicates that they did not transform into plerocercoids. Given an isometric body shape, this is more than 100 times the volume of procercoids grown in a good host environment (singly infected, adult females of M. albidus; Wedekind, 1997). In copepods, the procercoid growth rate is also thought to level off after the parasite has reached its asymptotic size in order to avoid killing the host (Michaud et al., 2006). Procercoids in culture, however, receive no feedback signals from the host, and perhaps therefore continue to grow.

The procercoids dissected from copepods did not seem to need a host specific stimulus to transform into plerocercoids in vitro. We cannot rule out completely that the high partial pressure of $\mathrm{CO}_{2}$ in the water, used to anaesthetize copepods before dissection had an influence. However, this seems unlikely, since no obvious difference was observed when using sterile filtered water instead of carbonated water.

The penetration process through the fish intestine might also require additional stimuli (Sukhdeo and Croll, 1981; Mulcahy et al., 2004) and the transformation was slow. Hence it is likely that the transformation process may be improved with some host specific stimuli. Exposure to $2 \%$ perch bile seemingly increase the speed of this process and moreover, also to transform cultured procercoids to early-stage plerocercoid larvae as those found as
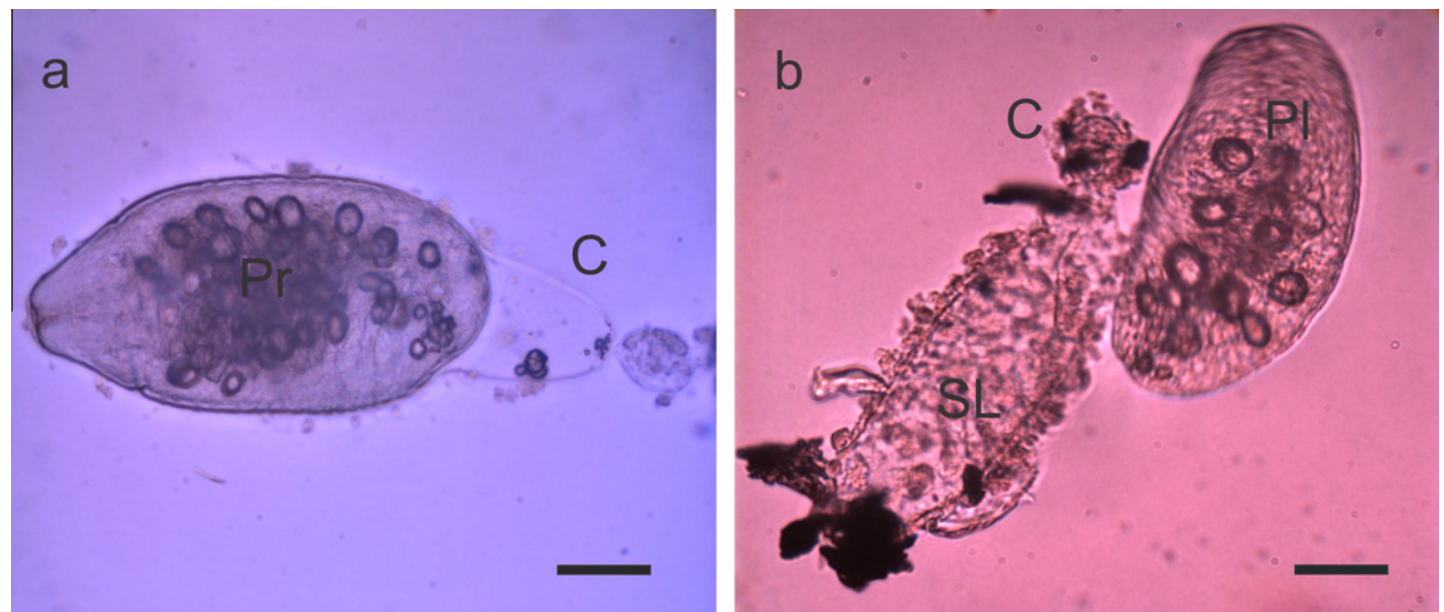

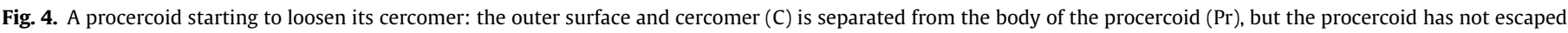
from the outer surface layer (a); and (b) a young plerocercoid ( $\mathrm{Pl})$ next to its surface layer (SL) and cercomer $(\mathrm{bar}=10 \mu \mathrm{m})$. 


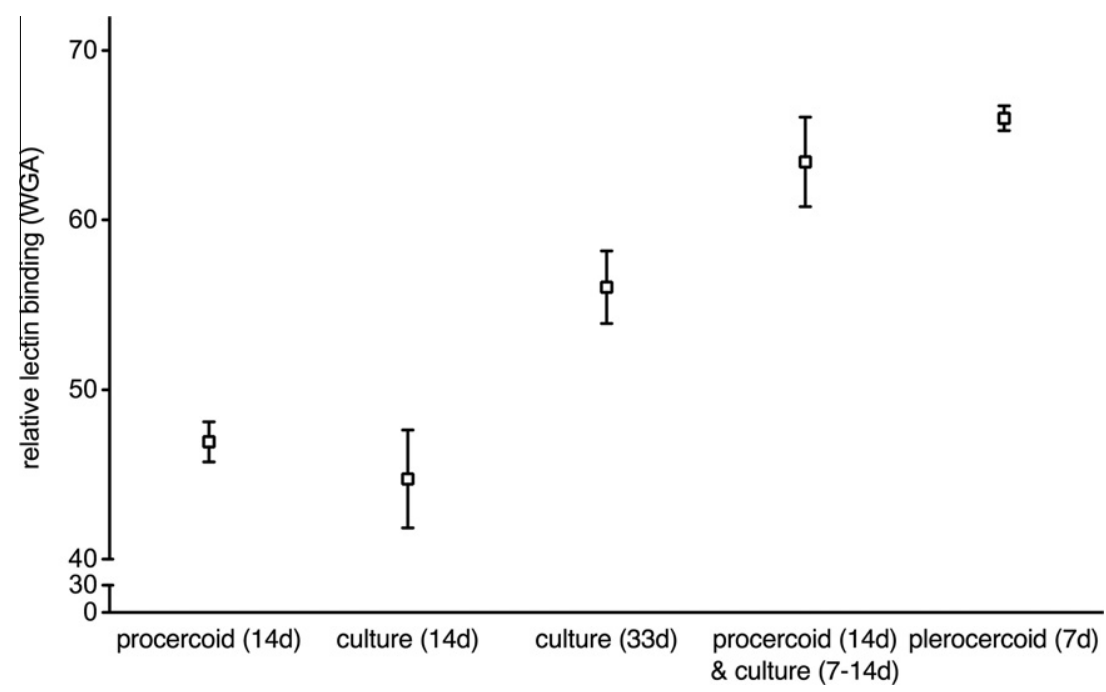

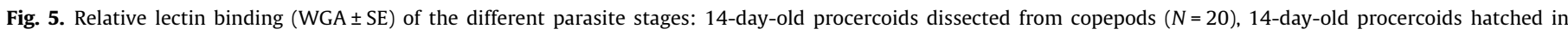

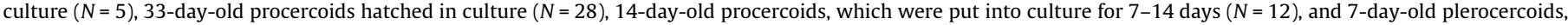
dissected from sticklebacks $(N=31)$.

the fish intestine penetrating stage (Hammerschmidt and Kurtz, 2007). Most likely also mechanical and enzymatic processes are important for getting through the fish intestine. Lack of some stimuli does, however, not imply that the procercoids had not undergone a full development to early-stage plerocercoids in the cultures. When transferred into the culture medium, their outer surface and cercomer started to loosen and the parasite increased their activity distinctly. Furthermore, the changes of the carbohydrate composition towards a plerocercoid-like surface layer, supports the assumption of a complete transformation. Additionally, Hammerschmidt and Kurtz (2007), observed that the cercomer occasionally was lost already in the frontal parts of the stickleback gut, while the penetration of the alimentary channel starts in the mid gut or further down in the intestine lumen, indicating that loss of the cercomer and penetration of the gut might be induced by different stimuli.

An important limitation in studying helminths is that long term culture conditions supporting the entire life-cycle is lagging behind (but see Smyth, 1990 and references therein; Coyne and Brake, 2001). In vitro cultivation of metazoan helminths has been proven to be a powerful tool within several disciplines such as immunology, evolution, ecology, endocrinology, sexual selection, behaviour, evo-devo and organismal parasitology (Smyth, 1990). Cultures are important because aspects of parasite ontogeny, behaviour tactics, and strategies may be confounded by various hosts' factors, as e.g. their immune system, resource constraints, within-host competition, host size, growth and life-histories, when bred in its natural hosts. It may also be difficult to track different development stages or transition processes of parasites within their hosts. Finally, the need of an in vitro culture is underscored, because the tapeworm S. solidus addressed here has developed into a natural model for studying the ecological and evolutionary questions of host-parasite interactions (Hammerschmidt and Kurtz, 2007 and reference therein) and complex life-cycles (Parker et al., 2003, 2009; Hammerschmidt et al., 2009). Hence, the system for an in vitro cultivation of S. solidus presented here will provide possibilities for experimental approaches to fundamental questions on the evolution of the fascinating and obviously successful life-cycles in parasitic helminths.

\section{Acknowledgments}

Thanks to Roswithe Derner for producing 27 different media with a smile on her face and Regina Leipnitz for culturing the copepods used in the experiments. Thanks to Daniel Benesh for advices and improvement of the English of the MS. This work has been done, when Per Jakobsen was on sabbatical leave. Per Jakobsen is grateful for the opportunities, financial support, help and good friendship he has received at the Max Planck Institute for Evolutionary Biology, Department of Evolutionary Ecology.

\section{References}

Arme, C., Owen, R.W., 1967. Infections of three-spined sticklebacks Gasterosteus aculeatus L. with plerocercoid larvae of Schistocephalus solidus (Müller 1776) with special preference to pathological effects. Parasitology 57, 301-307.

Barber, I., Arnott, S.A., 2000. Split-clutch IVF: a technique to examine indirect fitness consequences of mate preferences in sticklebacks. Behaviour 137, 1129-1140.

Barber, I., Huntingford, F.A., 1995. The effect of Schistocephalus solidus (Cestoda: Pseudophyllidea) on the foraging and shoaling behaviour of three-spined sticklebacks, Gasterosteus aculeatus. Behaviour 132, 1223-1240.

Barber, I., Scharsack, J.P., 2010. The three-spined stickleback -Schistocephalus solidus system: an experimental model for investigating host-parasite interactions in fish. Parasitology 137, 411-424.

Berntzen, A.K., Mueller, J.F., 1964. In vitro cultivation of Spirometra mansonoides (Cestoda) from the procercoid to the early adult. The Journal of Parasitology 50, 705-711.

Chervy, L., 2002. The terminology of larval cestodes or metacestodes. Systematic Parasitology 52, 1-33.

Clarke, A.S., 1954. Studies on the life cycle of the pseudophyllidean cestode Schistocephalus solidus. Proceedings of the Zoological Society of London 124, 257-302.

Coyne, C.P., Brake, D., 2001. Characterisation of Haemonchus contortus -derived cell populations propagated in vitro in a tissue culture environment and their potential to induce protective immunity in sheep. International Journal for Parasitology 31, 359-376.

Defaye, D., Such, J., Dussart, B., 1985. The alimentary canal of a fresh-water copepoda Macrocyclops albidus, and some other cyclopoida. Acta Zoologica 66, 119-129.

Dubinina, M.N., 1966. Tapeworms (Cestoda, Ligulidae) of the Fauna of the USSR. (Remnetsy (Cestoda, Lingulidae) Fauny SSSR). Nauka Publishers, Moscow.

Hammerschmidt, K., Kurtz, J., 2005a. Evolutionary implications of the adaptation to different immune systems in a parasite with a complex life cycle. Proceedings of the Royal Society of London B Biological Sciences 272, 2511-2518.

Hammerschmidt, K., Kurtz, J., 2005b. Surface carbohydrate composition of a tapeworm in its consecutive intermediate hosts: individual variation and fitness consequences. International Journal for Parasitology 35, 1499-1507.

Hammerschmidt, K., Kurtz, J., 2007. Schistocephalus solidus: establishment of tapeworms in sticklebacks - fast food or fast lane? Experimental Parasitology $116,142-149$.

Hammerschmidt, K., Kurtz, J., 2009. Ecological immunology of a tapeworms' interaction with its two consecutive hosts. Advances in Parasitology 68, 111137.

Hammerschmidt, K., Koch, K., Milinski, M., Chubb, J.C., Parker, G.A., 2009. When to go: optimization of host switching in parasites with complex life cycles. Evolution 63, 1976-1986. 
Hopkins, C.A., Smyth, J.D., 1951. Notes on the morphology and life history of Schistocephalus solidus (Cestoda, Diphyllobothriidae). Parasitology 41, 283-291.

Lüscher, A., Milinski, M., 2003. Simultaneous hermaphrodites reproducing in pairs self-fertilize some of their eggs: an experimental test of predictions of mixedmating and hermaphrodite's dilemma theory. Journal of Evolutionary Biology $16,1030-1037$

Michaud, M., Milinski, M., Parker, G.A., Chubb, J.C., 2006. Competitive growth strategies in intermediate hosts: experimental tests of a parasite life-history model using the cestode, Schistocephalus solidus. Evolutionary Ecology 20, 3957.

Milinski, M., 1985. Risk of predation of parasitized sticklebacks (Gasterosteus aculeatus L.) under competition for food. Behaviour 93, 203-216.

Milinski, M., 2006. Fitness consequences of selfing and outcrossing in the cestode Schistocephalus solidus. Integrative and Comparative Biology 46, 373-380.

Mulcahy, G., O’Neill, S., Donnelly, S., Dalton, J.P., 2004. Helminths at mucosa barriers- interaction with the immune system. Advanced Drug Delivery Reviews 56, 853-868.

Parker, G.A., Chubb, J.C., Ball, M.A., Roberts, G.N., 2003. Evolution of complex life cycles in helminth parasites. Nature 425, 480-484.

Parker, G.A., Ball, M.A., Chubb, J.C., Hammerschmidt, K., Milinski, M., 2009. When should a trophically transmitted parasite manipulate its host? Evolution 63 , 448-458.

Polzer, M., Conradt, U., 1994. Identification and partial characterization of the proteases from different developmental stages of Schistocephalus solidus (Cestoda: Pseudophyllida). International Journal of Parasitology 24, 967-973.
Pond, D.W., Harris, R.P., Brownlee, C., 1995. A microinjection technique using a pHsensitive dye to determine the gut $\mathrm{pH}$ of Calanus helgolandicus. Marine Biology 123, 75-79.

Sinha, D.P., Hopkins, C.A., 1967. Studies on Schistocephalus solidus: the effect of temperature on growth and maturation in vitro. Parasitology 57, 555-566.

Smyth, J.D., 1946. Studies on tapeworm physiology. I. The cultivation of Schistocephalus solidus in vitro. The Journal of Experimental Biology 23, 47-70.

Smyth, J.D., 1954. Studies on tapeworm physiology VII. Fertilization of Schistocephalus solidus in vitro. Experimental Parasitology 3, 64-71.

Smyth, J.D., 1959. Maturation of larval pseudophyllidean cestodes and strigeid trematodes under axenic conditions; the significance of nutritional levels in plathyhelminth development. Annuals of the New York Academy of Sciences $77,102-125$.

Smyth, J.D., 1990. In vitro cultivation of parasitic helminths. CRC Press, Boca Raton. Smyth, J.D., McManus, D.P., 1989. The physiology and biochemistry of cestodes. Cambridge University Press, Cambridge.

Sukhdeo, M.V., Croll, N.A., 1981. Gut propulsion in mice infected with Trichinella spiralis. Journal of Parasitology 67, 906-910.

van der Veen, I.T., Kurtz, J., 2002. To avoid or eliminate: cestode infections in copepods. Parasitology 124, 465-474.

Voge, M., Edmunds, H., 1969. Hatching in vitro of oncospheres from coracidia of Lacistarhyncus tenuis (Cestoda) Tetrarhyncidae. Journal of Parasitology 55, 571-573.

Wedekind, C., 1997. The infectivity, growth, and virulence of the cestode Schistocephalus solidus in its first intermediate host, the copepod Macrocyclops albidus. Parasitology 115, 317-324. 